\title{
Intraoperative Cardiac Injury, CTCAE
}

National Cancer Institute

\section{Source}

National Cancer Institute. Intraoperative Cardiac Injury, CT CAE. NCI Thesaurus. Code C143599.

A finding of damage to the heart during a surgical procedure. 